\title{
OPEN Nano-photoluminescence of natural anyon molecules and topological quantum computation
}

\author{
Alexander M. Mintairov ${ }^{1,2 \bowtie}$, Dmitrii V. Lebedev ${ }^{1}$, Alexei S. Vlasov ${ }^{1}$, Alexei O. Orlov $^{2}$, \\ Gregory L. Snider ${ }^{2}$ \& Steven A. Blundell ${ }^{3}$
}

The proposal of fault-tolerant quantum computations, which promise to dramatically improve the operation of quantum computers and to accelerate the development of the compact hardware for them, is based on topological quantum field theories, which rely on the existence in Nature of physical systems described by a Lagrangian containing a non-Abelian (NA) topological term. These are solidstate systems having two-dimensional electrons, which are coupled to magnetic-flux-quanta vortexes, forming complex particles, known as anyons. Topological quantum computing (TQC) operations thus represent a physical realization of the mathematical operations involving NA representations of a braid group $B_{n}$, generated by a set of $n$ localized anyons, which can be braided and fused using a "tweezer" and controlled by a detector. For most of the potential TOC material systems known so far, which are 2D-electron-gas semiconductor structure at high magnetic field and a variety of hybrid superconductor/topological-material heterostructures, the realization of anyon localization versus tweezing and detecting meets serious obstacles, chief among which are the necessity of using current control, i.e., mobile particles, of the TQC operations and high density electron puddles (containing thousands of electrons) to generate a single vortex. Here we demonstrate a novel system, in which these obstacles can be overcome, and in which vortexes are generated by a single electron. This is a $150 \mathrm{~nm}$ size many electron $\mathrm{InP} / \mathrm{GaInP}_{2}$ self-organized quantum dot, in which molecules, consisting of a few localized anyons, are naturally formed and exist at zero external magnetic field. We used high-spatial-resolution scanning magneto-photoluminescence spectroscopy measurements of a set of the dots having five and six electrons, together with many-body quantum mechanical calculations to demonstrate spontaneous formation of the anyon magneto-electron particles $\left(e^{\nu}\right)$ having fractional charge $v=n / k$, where $n=1-4$ and $k=3-15$ are the number of electrons and vortexes, respectively, arranged in molecular structures having a built-in (internal) magnetic field of 6-12 T. Using direct imaging of the molecular configurations we observed fusion and braiding of $e^{v}$-anyons under photoexcitation and revealed the possibility of using charge sensing for their control. Our investigations show that InP/Galn $P_{2}$ anyon-molecule $Q D s$, which have intrinsic transformations of localized $e^{v}$ anyons compatible with TQC operations and capable of being probed by charge sensing, are very promising for the realization of TQC.

Methods of topological quantum computation (TQC) are based on topological quantum field theories, which show that two-dimensional (2D) electron systems having magnetic-flux-vortexes can be modeled by a Hamiltonian whose eigenstates correspond to the states of quantum error correcting code ${ }^{1}$. This also means that they are described by an effective Lagrangian containing a non-Abelian (NA) topological term ${ }^{2}$. According to the theory, the quantum computation process in these systems will be protected from environmental distortion at the physical level and will be intrinsically fault-tolerant. Topological quantum-gate (TQG) processing represents a physical realization of mathematical operations involving NA representations of a braid group $B_{\mathrm{n}}$ generated by a set of $n$ localized particles (anyons) having zero-energy excitations, which are Majorana zero modes (MZMs),

${ }^{1}$ loffe Institute, Saint Petersburg 194021, Russia. ${ }^{2}$ Electrical Engineering, University of Notre Dame, Notre Dame, IN 46556, USA. ${ }^{3}$ University Grenoble Alpes, CEA, CNRS, IRIG, SyMMES, 38000 Grenoble, France. ${ }^{\square}$ email: amintairov@gmail.com 
each representing a qubit state. A MZM qubit involves magnetic-flux-vortexes having opposite directions, representing a particle-antiparticle pair. The operation of the gate includes moving particles around each other (braiding) and pairing (fusion) together with the generation of the particles and the control of the resulting state ${ }^{1,3}$. Up to now, all efforts to realize TQC have been focused on finding an appropriate system having anyons with $\mathrm{MZMs}^{4-9}$. Although it is not clear how the gate operation will be physically realized using the specific systems investigated ${ }^{4,10}$, it should generally involve some local potential perturbations for particle trapping and moving, i.e., "tweezing" 11-13. This tweezing procedure, changing the TQG qubit state, is the equivalent of the resonant electro-magnetic pulses that induce Rabi oscillations of a two-level qubit state in the conventional schemes of quantum computing $(\mathrm{QC})^{14}$. However, while the MZM-qubit and tweezing are supposed to be topologically protected, two-level qubits and Rabi oscillations are not, and they require adding redundant qubits to permit error-correcting code processing. This processing should provide an extremely low error-probability (fidelity) threshold to make QC operations fault-tolerant ${ }^{15}$. Such a threshold has been demonstrated only for a single qubit in the major QC platforms developed so far, which are Josephson tunnel junctions ${ }^{16}$ and electron-spin qubits in the different solid state environment or ion traps configuration ${ }^{17-21}$, but for two or more qubits the fidelity is poorer $^{22}$. Thus, the realization of TQC is of high demand.

TQC relies on the existence of anions, which are composite particles consisting of electrons and a few magnetic-flux-quanta vortexes ${ }^{23}$. The anyon has fractional charge and its wave-function can have an arbitrary phase after interchange. Multi-dimensional NA representations of $B_{n}$ are formed by a set of $n$ "coupled" anyons having a strongly degenerate ground state ${ }^{24}$. The wavefunction in this case is a vector depending on the position and the quantum number of each particle; particle exchange then gives a matrix, i.e. a NA transformation of this vector, which is topologically protected. Theoretically, NA anyons can be formed with half-vortexes in $p$-wave superconductors, which have MZM in the core ${ }^{25-27}$.

The richest and most investigated anyon system is a $2 \mathrm{D}$-electron semiconductor heterostructure in a perpendicular magnetic field in which anyon states are formed at fractional Landau level (LL) fillings and provide a dissipationless skipping-type edge current in conjunction with a transverse conductance plateau known as the fractional quantum Hall effect $(\mathrm{FQHE})^{28}$. More than $50 \mathrm{FQHE}$ anyon states were observed in the lowest Landau level (LLL) ${ }^{29}$ and about 14 states in the second Landau level (SLL) ${ }^{30}$. NA anyons have been suggested and probed for the state $v=5 / 2$ having half-filled $p$-wave state at SLL ${ }^{31-33}$. For the hypothetical SLL NA state $v=12 / 5$ the braids that yield a universal set of quantum gates were found ${ }^{3}$. FQHE anyons are probed by the edge currents involving hundreds of mobile particles, which make difficult direct TQC.

Another type of structures suggested for TQC are hybrid heterostructures, in which half-vortexes are expected to form using the proximity effect between a conventional $s$-wave superconductor and various topological structures/materials, such as semiconductor nanowires having strong a spin-orbit coupling ${ }^{5}$, chains of ferromagnetic atoms $s^{6}$, topological insulators ${ }^{7}$, anomalous Hall insulator-superconductor ${ }^{8}$, and an insulating $2 \mathrm{D}$ quantum magnet ${ }^{9}$. The key difference between these systems and the FQHE one is that a single vortex is formed by up to a few thousands of electrons forming normal core at the center of a superconducting current loop ${ }^{7}$, and because of this "massive" structure, tweezing seems to be hard to realize in these systems. The last two systems use edge currents and have the same difficulties as FQHE system.

Currently FQHE anyons are considered theoretically to be quasi-particle excitations of the host ground-state liquid ${ }^{34}$ and the quasi-particle picture is the basis for topological quantum field theories describing specific NA states and their use for building qubit and gate operations ${ }^{3,8}$. We have shown recently, however, that the FQHE anyon can exist as a single localized particles, which do not involve many-body interactions for their formation nor require quasi-particle concept for their description. Such fractionally charge particles were proposed to explain magneto-photoluminescence (magneto-PL) measurements of quasi-2D InP/GaInP $\mathrm{P}_{2}$ single-electron islands (quantum dots) having Wigner-Seitz radius $r_{\mathrm{s}} \sim 4{ }^{35}$. In this particle, which we called a magneto-electron $\left(e^{v}\right)$, a corresponding number of magnetic-quantum-flux vortexes $(k=1 / v)$ are self-generated.

Here we report the observation of molecular structures of $e^{v}$ s using magneto-PL measurements of QDs having about six-electrons and $r_{\mathrm{s}} \sim 2$ and show that these $e^{v}$-anyon molecules $\left(e^{v}\right.$-AM) represent a novel system, which can be used for the realization of TQGs. The measurements include the imaging of the emission area of individual PL lines, together with quantum mechanical calculations and analysis of their electronic structure in a magnetic field. Using these we demonstrate the self-formation of $e^{v}$-AMs and report observation of the $e^{v}$ AMs having $v \sim 3 / 5-1 / 4$, corresponding to a built-in magnetic field 6-12 T. We also observe a transformation of the $e^{v}$ arrangement in a $3 e^{2 / 7}$-AM under photo-excitation demonstrating fusion and braiding of the anyons. The observed transformation reveals a significant redistribution of the fractional charge within the dot, which suggests the use of single electron transistor charge sensing to control the TQC operations.

\section{Experimental details and data}

InP/Galn $\mathrm{P}_{2}$ quantum dots. We have studied nine single self-assembled $\mathrm{InP} / \mathrm{GaInP}_{2} \mathrm{QDs}^{36}$ having a lateral size of $D \sim 120 \mathrm{~nm}$ and five-to-six electrons using near-field scanning optical microscope (NSOM) at temperature $10 \mathrm{~K}$, external magnetic field range $B_{\mathrm{e}}=0-10 \mathrm{~T}$ and spatial resolution up to $25 \mathrm{~nm}$ (see "Methods").

The dots were selected using NSOM measurements of a set of about 200 dots in variety of InP/GaInP ${ }_{2}$ QDs structures in previous studies ${ }^{36-38}$. Specifically, nine dots investigated were obtained in the sample X3141 (see Ref. ${ }^{36}$ ) and all of them have "anomalous" PL spectral features revealed in Refs. ${ }^{36,37,39}$ in comparison with the "normal" dots typically having more electrons and smaller size (see below). Two of such dots, also discussed here, were measured in the same sample.

All dots (except one normal) are denoted by $\mathrm{D} k x$, where the letter $\mathrm{D}$ indicates the initial identification of the shell filling (see the dot D5 in Ref. ${ }^{36}$, which is labeled D4e dot here), $k$ enumerates the dots and $x=\mathrm{a}, \mathrm{b}, \mathrm{c} \ldots$ 


\begin{tabular}{|l|l|l|l|l|l|l|l|l|l|}
\hline$\#$ & Name & AM type & $\boldsymbol{E}_{\mathbf{0}}(\mathbf{e V})$ & $\boldsymbol{\Delta} \boldsymbol{E}_{\boldsymbol{s p}}(\mathbf{m e V})$ & $\boldsymbol{D}(\mathbf{n m})$ & $\boldsymbol{r}_{\mathrm{s}}$ & $\boldsymbol{B}_{\mathrm{bi}}(\mathrm{T})$ & $\boldsymbol{v}$ & $\boldsymbol{e}^{\boldsymbol{v}}$ molecular state PS(IS) \\
\hline 1 & $\mathrm{D} 2 \mathrm{~b}$ & $\mathrm{AM}_{\mathrm{m}}$ & 1.7063 & 1.9 & $\sim 110$ & 2.3 & 9 & $1 / 3$ & $6 e^{1 / 3}$ \\
\hline 2 & $\mathrm{D} 4 \mathrm{~b}$ & $\mathrm{AM}_{\mathrm{m}}$ & 1.6870 & 1.7 & $\sim 120$ & 2.35 & 11 & $2 / 7$ & $3 e^{2 / 7}$ \\
\hline 3 & $\mathrm{D} 5 \mathrm{~b}$ & $\mathrm{AM}_{6,0}$ & 1.7143 & 1.7 & $\sim 130$ & 2.4 & 11 & $3 / 11$ & $2 e^{3 / 11}$ \\
\hline 4 & $\mathrm{D} 1 \mathrm{~d}$ & $\mathrm{AM}_{5,1}$ & 1.7075 & 2.0 & $\sim 110$ & 2.2 & 6 & $3 / 5$ & $2 e^{3 / 5}$ \\
\hline 5 & $\mathrm{D} 5 \mathrm{~d}$ & $\mathrm{AM}_{5,1}$ & 1.7043 & 2.0 & $\sim 110$ & 2.2 & 6 & $3 / 5$ & $6 e^{1 / 3}$ \\
\hline 6 & $\mathrm{D} 1 \mathrm{e}$ & $\mathrm{AM}_{6,0}$ & 1.7686 & 1.7 & $150 / 70^{\mathrm{a}}$ & 2.4 & 11 & $4 / 15$ & $3 e^{1 / 3}+e^{4 / 15}\left(3 e^{2 / 7}\right)$ \\
\hline 7 & $\mathrm{D} 2 \mathrm{e}$ & $\mathrm{AM}_{\mathrm{m}}$ & 1.7293 & 1.4 & $\sim 140$ & 2.6 & 12 & $3 / 13$ & $2 e^{3 / 14}$ \\
\hline 8 & $\mathrm{D} 4 \mathrm{e}$ & $\mathrm{AM}_{6,0}$ & 1.7311 & 1.6 & $120 / 70$ & 2.5 & 12 & $1 / 4$ & $6 e^{1 / 4}\left(5 e^{1 / 4}\right)$ \\
\hline 9 & $\mathrm{D} 5 \mathrm{e}$ & $\mathrm{AM}_{\mathrm{m}}$ & 1.7913 & 1.6 & $\sim 135$ & 2.5 & 12 & $1 / 4$ & $6 e^{1 / 4}$ \\
\hline
\end{tabular}

Table 1. Parameters of $\mathrm{AM}$ states of $\mathrm{InP} / \mathrm{GaInP}_{2}$ QDs measured using NSOM.

indicates the NSOM run. One normal dot is denoted F1e. The dots are located within an area $\sim 2 \times 2 \mu \mathrm{m}^{2}$ for the same run/scan (see below) and within an area $\sim 2 \times 2 \mathrm{~mm}^{2}$ for different runs.

For anomalous dots the parameters measured include the AM type, the main peak energy $E_{0}$, the $s-p$ splitting $\Delta E_{\mathrm{sp}}$, the size $D$, the Wigner-Seitz radius $r_{\mathrm{s}}$, the built-in magnetic field $B_{\mathrm{bi}}, v$ and the $e^{v}$ configuration. The AM type, $E_{0}$ and $\Delta E_{\mathrm{sp}}$, were measured from intensity distribution, position and energy splitting of the PL spectral components. The $D$ values for most of the dots were measured/estimated directly from scanning experiments and used for calculation of $r_{\mathrm{s}}=D N^{-0.5} /\left(2 a_{B}{ }^{*}\right)$, where $a_{B}{ }^{*} \sim 8 \mathrm{~nm}^{35} . B_{\mathrm{bi}}$ and $v$ was estimated from a complex analysis of the whole data set including the dependence of the PL spectra on $B_{\mathrm{e}}$, the NSOM maps and theoretical calculations/ analysis (see below). The $e^{v}$ configuration was suggested from analysis of the whole data set. These parameters apply to the photo-excited state (PS), which for all dots (except D1e) have $N^{*}=N+1=6$ electrons, where $N$ is number of electrons in initial (IS) state. For D1e $N^{*}=7$.

Table 1 summarizes the dot parameters measured. The parameters, important for further discussion, are $r_{s}$, which changes from 2.2 to 2.6 making up $20 \%$ variations and $B_{\mathrm{bi}}$, which changes from 6 to 12 T making up two times $(200 \%)$ variations.

Theoretical description. The analysis of the experimental data was done using a phenomenological description within a framework of a general theory based on exact quantum mechanical calculations involving Fock-Darwin (FD), Hartree-Fock (HF) and configuration interaction (CI) approaches, developed for a few 2D electrons confined in circular potential in a perpendicular magnetic field about 20 years ago ${ }^{40-42}$.

The many-body HF and CI methods were used to calculate dependence of the energy structure together with the shell energy and electron distribution on magnetic field for specific QD studied taking into account their shape, and, thus, a circular symmetry breaking effects (see "Methods"). These were used for analysis and comparison with corresponding experimental data.

Single-particle FD spectrum for circular symmetric $\operatorname{dots}^{43}$ was used in the form $E_{k, l}(B)=E_{k, l}\left(B_{\mathrm{e}}+B_{\mathrm{bi}}\right)$, where $E_{k, l}(B)=E_{k, l}{ }^{\mathrm{FD}}(\Omega)=\hbar \Omega(k+1)-1 / 2 \hbar \omega_{\mathrm{c}} l$, and $\Omega^{2}=\omega_{0}{ }^{2}+\omega_{\mathrm{c}}{ }^{2} / 4$, where $\omega_{0}$ and $\omega_{\mathrm{c}}=e^{*} B / m^{*}$ are the quantum confinement and cyclotron frequencies, respectively, and $k$ and $l$ are the principal $(k=0,1, .$.$) and azimuthal (l=-k,-k+2$, $\ldots, k$-2) quantum numbers. We used an approximate expression for $E_{0,0}\left(B_{0}\right) \approx N^{\star} V_{\mathrm{c}}$ derived in Ref. ${ }^{38}$ for $N=1$, where $v_{\mathrm{c}=} 0.07 \omega_{\mathrm{c}}\left(m^{*} \Omega\right)^{-0.5}$ is a matrix element of the Coulomb interaction.

The FD spectrum was used to fit the experimental shifts of PL lines versus external magnetic field $B_{\mathrm{e}}$ to estimate the electron charge $e^{\star}$ and $B_{\mathrm{bi}}$.

Photo-luminescence spectra. Anomalous spectral features and intensity distributions. The spectra of anomalous dots presented in Fig. 1a have a set of sharp peaks, which are a main zero-energy $e_{0}$-peak and about five $e_{1}-e_{5}$ peaks, related to anti-Stokes components (ASCs) having a splitting of $\sim 0.5 \mathrm{meV}$. This is significantly different from the spectra of normal dots presented in the insert (see figure caption for their parameters), which reveal two or three ASC peaks, a few times larger splitting (3-5 meV), and order-of-magnitude larger peak broadening. While, in the normal dots, the zero-energy and ASCs peaks are related to the occupied degenerate $s-p-, d-, \ldots$ electron (e) shells (including a photo-excited $e$ ), in the anomalous ones they are related to single spin-polarized es and manifest $B_{\mathrm{bi}}$, as we will show below. The energy splitting between the $e_{0}$ and the $e_{3}$ peaks $\Delta E_{\mathrm{sp}}$, shown by a horizontal arrow, is related to the $s$ - $p$-shell splitting, which, according to HF calculations, determines a quantum confinement energy $\hbar \omega_{0}{ }^{*}=0.7 \Delta E_{\mathrm{sp}}$. Besides the ASCs, Stokes components (SCs) denoted as $n w_{0}\left(n=1-3, \hbar \omega_{0} \approx \Delta E_{\mathrm{sp}}\right)$ are observed for some dots (D1d, D2b, D4e and D1e) and they are related to a centerof-mass vibration.

While the anomalous spectral features are nearly the same for all dots, the intensity distribution of ASCs shows significant variations. These variations reveal three types denoted by $\mathrm{AM}_{5,1}, \mathrm{AM}_{\mathrm{m}}$ and $\mathrm{AM}_{6,0}$. For the $\mathrm{AM}_{5,1}$ type (see two lowest spectra in Fig. 1a) the ASCs are an order of magnitude weaker than $e_{0}$. For the $\mathrm{AM}_{\mathrm{m}}$ type (four middle spectra) the intensity of the ASCs increases a few times reaching a value of up to half of $e_{0}$. For the $\mathrm{AM}_{6,0}$ type (see three upper spectra) the intensity of the ASC peaks increases further and becomes nearly the same as the intensity of the peak $e_{0}$. 

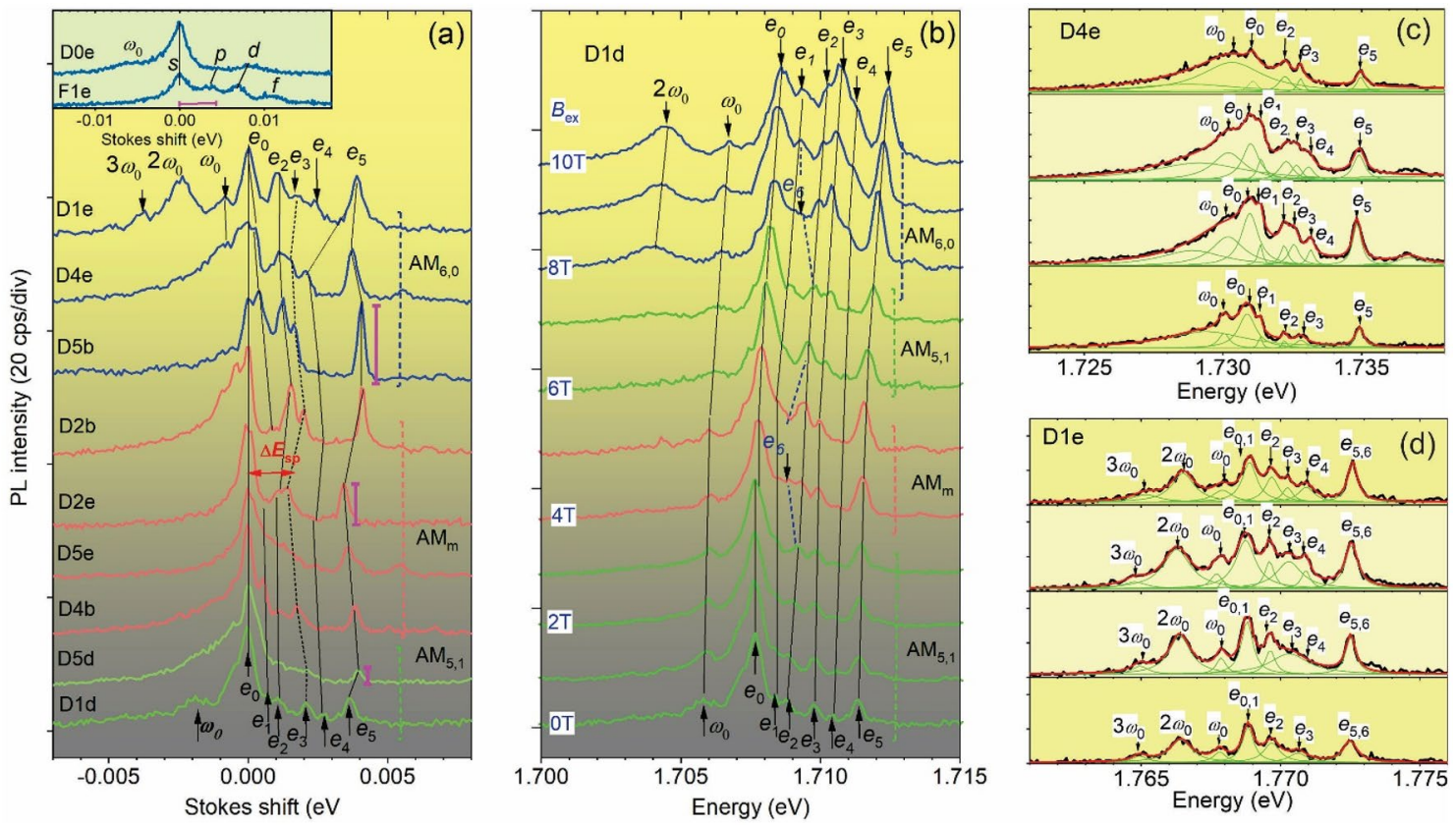

Figure 1. PL spectra of $\mathrm{D} k x$ anomalous $\mathrm{InP} / \mathrm{GaInP}_{2} \mathrm{QDs}$ at zero external magnetic field $B_{\mathrm{e}}$. Solid lines connect the corresponding PL peaks, dashed ones connect peak $e_{3}$. The upper insert shows PL spectra of two normal dots D0e $\left(N \sim 8, \Delta E_{\mathrm{sp}} \sim 4.5 \mathrm{meV}\right)$ and $\mathrm{F} 1 \mathrm{e}\left(N \sim 12, \Delta E_{\mathrm{sp}} \sim 3.5 \mathrm{meV}\right)$ and the horizontal bar indicates the spectral range of anomalous dots (a). PL spectra of the QD D1d in external magnetic fields $B_{\mathrm{e}}=0,1,2 \ldots$ and $10 \mathrm{~T}$ (b). Spatially resolved NSOM spectra $\left(B_{\mathrm{e}}=0 \mathrm{~T}\right)$ of D4e (c) and D1e (d) QDs, respectively, taken at tip positions separated by $50 \mathrm{~nm}$.

Magnetic field dependence. In the magnetic fields $B_{\mathrm{e}}=0-10 \mathrm{~T}$ (see Fig. 1b) the $\mathrm{AM}_{5,1}$-type D1d dot reveals a very weak diamagnetic shifts of PL spectral lines (about $0.5 \mathrm{meV}$ for $10 \mathrm{~T}$ ) and a strong change in relative intensity versus $B_{\mathrm{e}}$ resulting in the appearance of the type $\mathrm{AM}_{\mathrm{m}}$ at $B_{\mathrm{e}}=4-5 \mathrm{~T}$ and type $\mathrm{AM}_{6,0}$ at $8-10 \mathrm{~T}$. The emergence of these two types is accompanied by the appearance of an additional peak $e_{6}$ between peaks $e_{1}$ and $e_{2}$. For the $\mathrm{AM}_{6,0}$ type a SC at $2 \omega_{0}$ appears, similar to the dot D1e (see Fig. 1a).

For normal dots the shifts (not shown here) are an order of magnitude stronger (up to $1 \mathrm{meV} / \mathrm{T}$ ) having anti-crossings and paramagnetic regions at fields $<2 \mathrm{~T}$, while the intensity distributions do not show significant changes, as will be discussed elsewhere.

Spatially-resolve spectra and imaging. The dots reveal anomalous size of the emission area (EA) of the single- $e$ PL peaks, which is seen from spatially resolved data for dots D1e and D4e (see Fig. 1c,d and Fig. 2a-e). A variation of the intensity of $e_{0}-e_{5}$ peaks down to $\sim 0.4 \%$ per $\mathrm{nm}$ is seen in spectra in Fig. $1 \mathrm{c}$, d. This corresponds to an EA size $d_{\mathrm{EA}}$ down to $\sim 25 \mathrm{~nm}$ as it is seen in the PL intensity maps in Fig. 2a,b, giving $d_{\mathrm{EA}}=40 \pm 20 \mathrm{~nm}$.

The maps also show the location of the individual es, and the combined maps presented in Fig. 2c,d display molecular structures. The molecular structure is absent in the normal D0e and F1e dots, the EA of which corresponds to the dot size of $\sim 90$ and $110 \mathrm{~nm}$, respectively. This is seen in the combined map of the area containing these four dots and presented in Fig. 2e. In the map the images of IS are presented for D1e and D4e dots. The map shows that these are neighboring dots arranged in a liner chain with separation $\sim 200 \mathrm{~nm}$.

The molecular structure of the QD D4e in PS in Fig. 2c is a compressed hexagon having a size $\sim 120 \times 70 \mathrm{~nm}$ and a bond length $\sim 40 \mathrm{~nm}$. The IS structure (see Fig. 2e) is a pentagon, in which $e_{0}$ and $e_{1}$ electrons are shifted by 10-20 nm along the $(x, y)$ diagonal, as follows from $\omega_{0}$ EAs shape.

A complicated quasi-1D molecular structure oriented along the $(x, y)$-diagonal and having a size $\sim 150 \times 70$ $\mathrm{nm}^{2}$ is observed for D1e (see Fig. $2 \mathrm{~d}$ ). In this structure $e_{1}, e_{2}, e_{3}$ and $e_{6}$ EAs overlap and $e_{0,1}$ and $e_{5,6}$ EAs have two locations, revealing degenerate $2 e$ states, which gives a $7 e$ PS of this dot. The overlapped peaks are located in the upper right corner of the map; the rest three peaks are located along the $(x, y)$-diagonal below at the distance $\sim 70 \mathrm{~nm}$ and separated from each other by $40 \mathrm{~nm}$.

The SCs maps correspond to a $\sim 60 \mathrm{~nm}$ vertical up shift of $e_{4}\left(\omega_{0}\right.$ map), a $\sim 60 \mathrm{~nm}$ down shift $e_{3}\left(2 \omega_{0}\right.$ map) and a $\sim 40 \mathrm{~nm}$ down shift of $e_{5}$ along the $(x,-y)$-diagonal ( $2 \omega_{0}$ map). This results in $e_{0}-e_{4}$ and $e_{3}-e_{5}$ pairing and in the IS the molecular structure has nearly equilateral triangle arrangement of paired es at the vertexes, having sides $\sim 80 \mathrm{~nm}$ and $90 \mathrm{~nm}$ and a bond length $\sim 60 \mathrm{~nm}$.

In the combined maps in Fig. 2c,d a dark-light color code of EAs corresponds to large-small separation of $e s$ from the photo-excited hole, which allows determine its location as shown in the maps. Thus, specific spectral shape observed is related to a specific distribution of such separations (see below).

Analysis of the data. Classification of energies and states in magnetic field. A general theory of circular dots having $N$ electrons in a magnetic field distinguishes four contributions to the total energy $E_{\text {tot }}$, first 

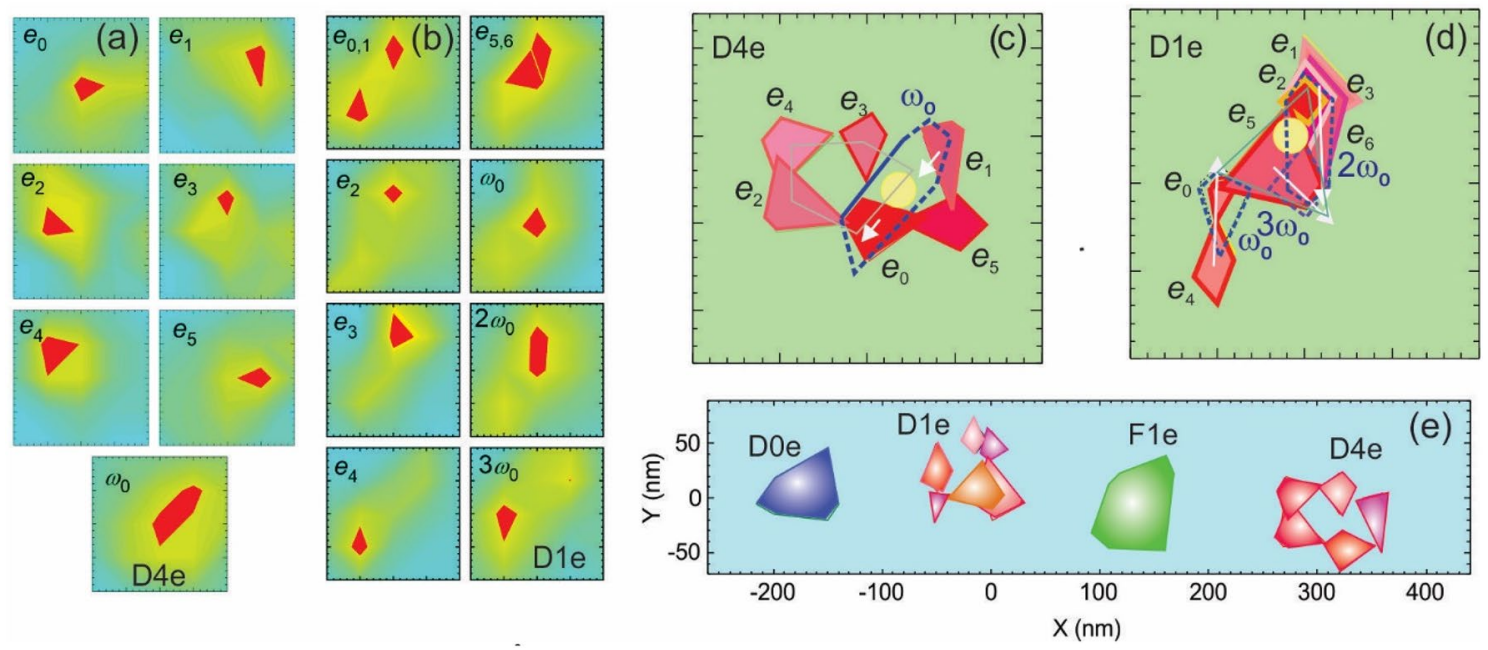

Figure 2. NSOM intensity images (size $200 \times 200 \mathrm{~nm}^{2}$ ) of the individual spectral lines of D4e (a) and D1e (b) QDs, respectively. Combined EAs of a and b, respectively $(\mathbf{c}, \mathbf{d})$. Solid shapes and dashed contours are ASCs and SCs, respectively. Arrows show the shift of the ASC EAs in the IS and a circle marks the expected location of a photo-excited hole. Dark-light color code of ASCs outlines line intensity. Combined maps of D0e, D1e, F1e and D4e in IS (e).

discussed for $2 e$ in Ref. ${ }^{4}$, which are the kinetic energies of localization induced by the confinement potential $E_{\text {conf }}(N)$ and by the magnetic field, i.e., cyclotron motion, $E_{\text {cycl }}(N, B)$, respectively, and the Coulomb energies of electrons center-of-mass $E_{\text {Coul,c.m }}(N)$ and relative $E_{\text {Coul,rel }}(N, B)$ motion, respectively. The $B$-independent parts are $E_{\text {conf }}(N)=K(N) \hbar \omega_{0}^{*}$ and $E_{\text {Coul,c.m }}(N) \quad r_{\mathrm{s}} E_{\text {conf }}(N)$. The $B$-dependent cyclotron term $E_{\text {cycl }}(N, B)=\hbar \omega_{\mathrm{c}} N^{\star} / 2$ is dominant for large fields when $\omega_{\mathrm{c}}>\omega_{0}$. The $B$-dependent term $E_{\text {Coul,rel }}(N, B)$ is non-trivial and, in spite of its extremely small value $\sim 0.01 E_{\text {Coul,c.m }}$ (see Fig. $3 \mathrm{~d}$ below), provides ground-state transitions having discrete total angular momentum values $L_{z}(B)=\sum_{i}^{N} l_{z i}$, where $l_{\mathrm{zi}}$ is the angular momentum of single-es, known as magic numbers $\left(L_{z}{ }^{\mathrm{MN}}\right)$. These appear because of the necessity of matching the spatial structure of the $e$ wave-functions and the spatial symmetry of the $e$ arrangement ${ }^{45}$. The $e$ state of the specific $L_{z}{ }^{\mathrm{MN}}$ is related to a filling factor $v=L_{0} / L_{\mathrm{z}}{ }^{\mathrm{MN}}$, where $L_{0}=N(N-1) / 2$, linking it to $\mathrm{FQHE}^{46}$. The set of values $B\left(L_{\mathrm{z}}^{\mathrm{MN}}\right)=B_{v}$ represent a "spectrum", which for specific $N$ value, is scaled as $\sim 1 / r_{\mathrm{s}}$.

For a circular symmetry analog of the dot D1d, for which $E_{\text {tot }}(0)=90 \mathrm{meV}, E_{\text {cycl }}(B)=4.2 / B(\mathrm{meV} / \mathrm{T})$ and $\Delta L_{z}=5$ or $6^{46}$, respectively, the molecular states are spin-polarized, $v<1$-states and are developed at $B>4 \mathrm{~T}$, after maximum density droplet formation ${ }^{47}$. In the range $B=5-14 \mathrm{~T}$, embracing $v=1 / 2-3 / 10$, the dominant configuration is $(5,1)^{46}$.

Molecular configurations and PL spectra intensity distributions. In the QD D1d the mixing of the configurations takes place, due to non-circular shape, as is seen in electron density distributions, calculated using CI, in Fig. 3a. Nine density distributions shown for $v$ from 1 to 3/14 occupying $B$ range 2.7-16 T reveal the onset of molecular structure formation near $v=5 / 7(B \sim 5 \mathrm{~T})$ similar to the circular symmetry. A "pure" $(6,0)$ and $(5,1)$ configurations appear for $v=5 / 7$ and $1 / 4$ and for $v=2 / 7$ and 3/14, respectively. For other values of $v$ two mixed configurations are appeared. In the one (see $v=1 / 2$ ) a central $e$ maximum is shifted $\sim 10-20 \mathrm{~nm}$ down from the center, and in the other (see $v=1 / 3$ ) a pentagon with adjacent single-e maxima at the left is formed. Note, that at $v=1 / 4$ the calculated configuration is a compressed hexagon, exactly the same as observed experimentally in D1e, which implies $B_{\mathrm{bi}} \sim 12 \mathrm{~T}$.

Molecular configurations in Fig. 3a can be assigned to specific AM types spectra observed in Fig. 1a,b. They, together with the maps in Fig. 2c,d, show that the appearance of different AM types spectra reflects a competition between $(6,0)$ and $(5,1)$ isomers for a non-circular dot shape and different $B_{\mathrm{bi}}$ or $B$. In these the former has nearly equal $\mathrm{AM}_{6,0}$ type ASC intensity, due to the nearly equal separation of electrons from the hole, while the latter has one dominant peak $\mathrm{AM}_{5,1}$ type owing to its location closer to the hole and the $\mathrm{AM}_{\mathrm{m}}$-type has intermediate, mixed configurations and intensity distributions.

Magneto-electrons and spontaneous anyon molecule formation. The analysis of the calculated $B$-dispersion of HF and FD shell energies, $L_{z}, E_{\text {tot }}$ and their comparison with experimental data of the dot D1d shown in Fig. $3 \mathrm{~b}$ together with the FD fit to these data in Fig. $3 \mathrm{c}$ and $E_{\text {Coul,rel }}$ and $E_{\text {tot }}$, shown in Figs. 3d,e, respectively, reveal the formation of the fractionally charged $e^{v}$.

The $E_{\text {tot }}$ curve in Fig. $3 \mathrm{~b}$ shows a nearly dispersionless $E_{\text {conf }}(N)+E_{\text {Coul,c.m }}(N)$ contribution for $B<3$ T, a linear increase from $E_{\text {cycl }}(N, B)$ and $B>4 \mathrm{~T}$, and weak oscillations from $E_{\text {Coul,rel }}(B)$ at $B_{v}$ (see also plot of $E_{\text {Coul,rel }}(B)$ in Fig. 3d) over the entire $B$-range. The $L_{z}{ }^{\mathrm{MN}}$ states of the dot D1d are clearly visible on $L_{z}(B)$ curve as plateaus and weak kinks at $L_{z}=5,9,15,21,2530,35$ and 45 . The corresponding $B_{v}$-spectrum has a $v$ values set from 3 to $1 / 3$ and is very close to that of circular $\operatorname{dot}^{46}$. The plateaus are also seen in the region $v>1$ corresponding to 

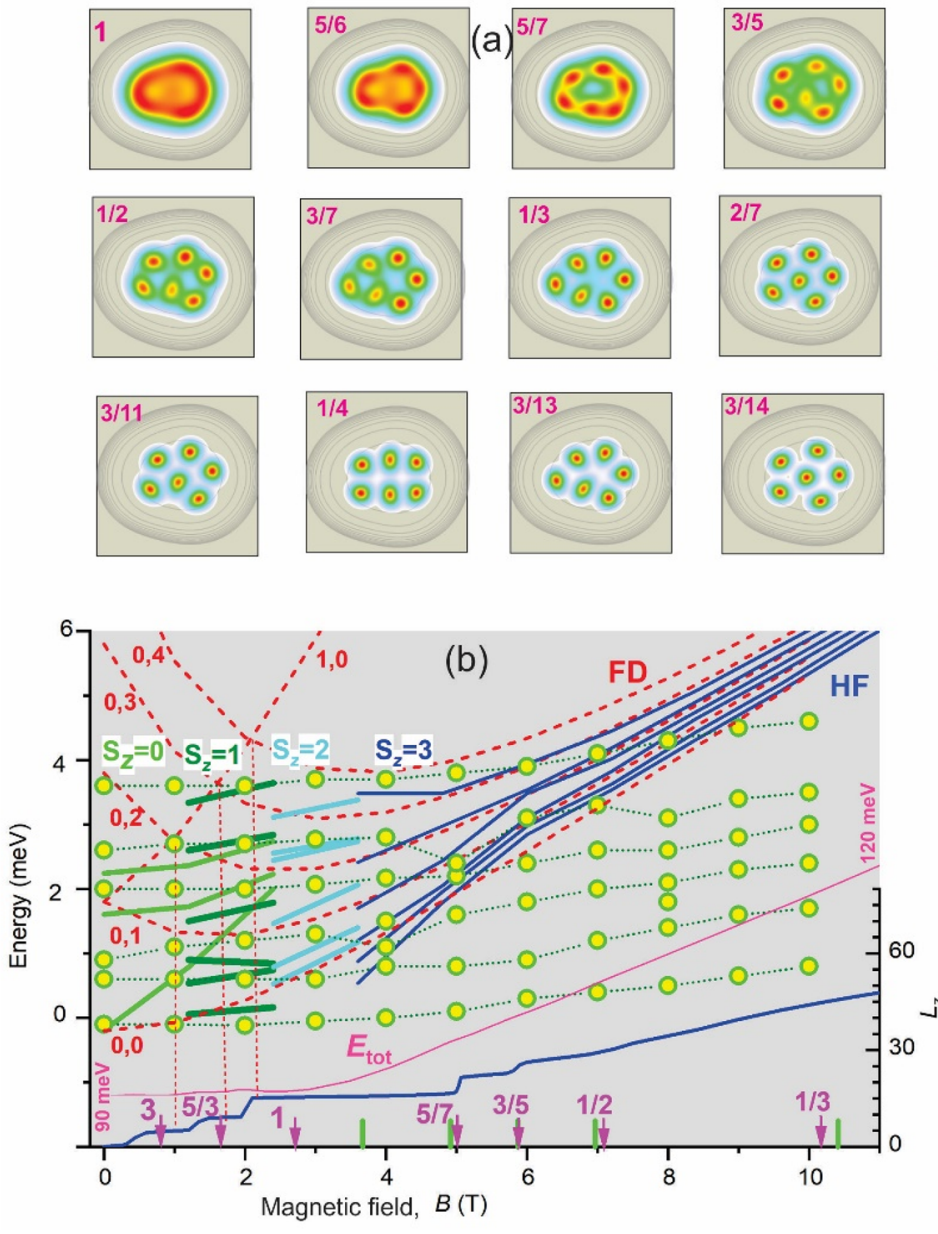

Figure 3. Calculated electron density distributions given by CI of the QD D1d for filling factors (numbers in the plots) $v=1,5 / 6,5 / 7,3 / 5,1 / 2,3 / 7,1 / 3,2 / 7,3 / 11,1 / 4,3 / 13$ and $3 / 14$. Contours indicate the deformed confinement potential (a). Calculated total energy (thin line) ranging from $90 \mathrm{meV}$ at $\mathrm{B}=0 \mathrm{~T}$ to $120 \mathrm{meV}$ at $\mathrm{B}=12 \mathrm{~T}$, total angular momentum $L_{z}$ (thick line, axis on right side) and shifts given by FD (dashed lines) and by $\mathrm{HF}_{\mathrm{z}}=0-3$ (solid lines) versus a magnetic field range 0-10 T. Vertical dashed lines mark the crossing of the 1,0 and the $0, l$ FD levels. Solid circles are the experimental shifts of ASCs. The vertical arrows and bars on $B$-axis are $B_{v}$ "spectrum" of non-circular and circular dot (see text), respectively (b). PL spectra (contour plot) and peak shifts (open circles) versus total field $B$ and FD fit (curves) $(\mathbf{c}) . E_{\text {Coul,rel }}(B)(\mathbf{d}) . e$ versus $e^{v} E_{\mathrm{tot}}(B)$ and vortexes occupying single- $e$ area (circles) for $v=1 / 2,1 / 3$ and $1 / 4$ (e). Schematic $e^{v}$ molecular arrangement for the QDs D4d, D1e and De4 having $v=3 / 5,2 / 7$ and $1 / 4$, respectively (f).

the integer $(v=3)$ and SLL fractional $(v=5 / 3)$ QHE states, demonstrating its deep connection to the localized es states in QDs.

This connection is also revealed for FD states, which appears as a matching of the crossings between the $(1,0)$ and $(0, l)$ FD levels to $L_{z}{ }^{\mathrm{MN}}$ transitions at $v=3$ to $5 / 3$ and to 1 , corresponding to a total spin transitions from $S_{z}=0$ to 1 to 2 . The HF energies, accounting for spin and $p_{x}-p_{y}$ circular symmetry distortion splittings, are shown in the figure in corresponding regions and approximately match the FD energies, neglecting the splitting. Both HF and FD energies at large fields come out to the LLL line $\hbar \omega_{\mathrm{c}} / 2$, with a nearly order-of-magnitude reduction of inter level splitting, which evolves to zero in the limit $B \rightarrow \infty$.

From Fig. $3 \mathrm{~b}$ we can see, that the critical discrepancy between the calculated FD/HF energies and the measured ASC shifts is that the latter have negligible $B$-dispersion and an order of magnitude larger level splitting over the entire 0-10 T magnetic field range. This implies a reduction of $\omega_{c}$, as can be revealed from the FD fit, shown in Fig. 3c. The FD fit gives a general matching with experimental shifts and splittings of $e_{0}-e_{5}$ peaks for $B_{\mathrm{bi}} \sim 6 \mathrm{~T}$ and a three-fold reduction of $\omega_{\mathrm{c}}$. The total internal field $B=B_{\mathrm{bi}}+B_{\mathrm{e}}$ thus has the range $B=6-16 \mathrm{~T}$, which includes $v=1 / 2$ and $1 / 4$.

The matching, however, does not account for the appearance of the additional peak $e_{6}$ and the $\sim 0.2 \mathrm{meV}$ variations of the experimental $B$-dispersion, taking place near $B \sim 7,10$ and $14 \mathrm{~T}$. These features correspond to the peculiarities of $E_{\text {Coul,rel }}(B)$ in Fig. $3 \mathrm{~d}$ at $v=1 / 2,1 / 3,2 / 7$ and $1 / 4$ and, thus, can be interpreted as the experimental signatures of these states. The appearance of the $e_{6}$ peak could indicate crossing of single-e $l_{\mathrm{z}}$ levels near steps in $L_{z}{ }^{\mathrm{MN}}$. 

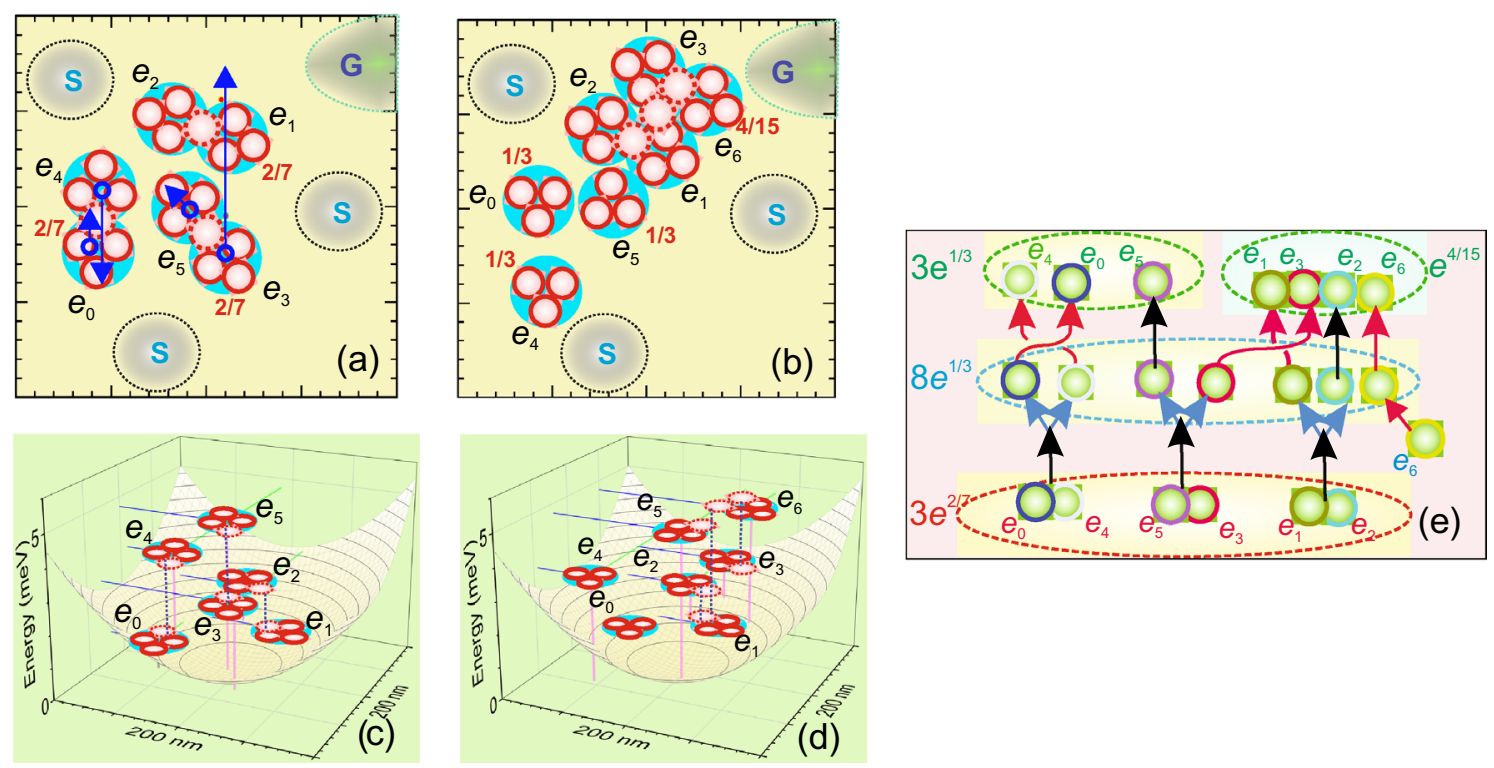

Figure 4. Arrangement of $e^{v} \mathrm{~s}$ (large/small circles are electrons/vortexes, dashed circles are coupling vortexes, numbers are fractional charge) in $6 e$-IS (a) and 7e-PS (b) of the dot D1e, respectively and adjacent nanocircuit elements (charge sensors $\mathrm{S}$ and gate electrode $\mathrm{G}$ ). Arrows are displacement vectors of corresponding $e^{v} \mathrm{~s}$, respectively. Energy-space diagrams overlaid on illustrative parabolic confinement potential, respectively, (c, d) . Dashed vertical lines outline $e^{v}$ coupling. Diagram of world lines of $B_{7}$-group describing a PL process of D1e QD (e).

Reduction of $\omega_{\mathrm{c}}$ can be interpreted as fractional charge and manifests a self-generation of the magneticquanta-flux vortexes by single es forming $e^{v} \mathrm{~s}^{35}$. The vortex self-generation arises because of the reduction of $E_{\text {tot }}$ parts $E_{\text {Coul,c.m }}+E_{\text {cycl }}$ caused by the decrease in charge and the dissipationless (superconducting) motion of es occupying quantum confined states for $r_{\mathrm{s}}>2$. Such a reduction of $E_{\mathrm{tot}}$ for $e^{v} \mathrm{~s}$ compared to es is shown in Fig. 3e. It reveals a $\sim 10 \mathrm{meV}$ minimum at $v=5 / 3$ and a gradual decrease from $100 \mathrm{meV}$ for $v \sim 5 / 7$ to $50 \mathrm{meV}$ for $v \sim 1 / 4$, which corresponds to increase of the energy drop from 10 to $80 \mathrm{meV}$.

Size dependence of molecular structure. Since the vortexes have fixed radius $\sim a_{B}^{* 35}$, the resulting charge, i.e., $v, B_{\mathrm{bi}}$, and AM configuration, are determined mostly by $r_{\mathrm{s}}$. The size of the $e^{v}$ area $d_{v}$ increases by a fraction $\sim 0.1$ per vortex, as can be found from simple geometrical drawing (see the corresponding cartoons for $v=1 / 2,1 / 3$ and $1 / 4 e^{v}$ areas in Fig. 3e). The $d_{v}$ value of $\sim 40 \mathrm{~nm}$ comes from the calculations for $v=1 / 4$ is in agreement with the measured $d_{\mathrm{EA}}$ in the dot D4e. Thus, this dot can be considered as $6 e^{1 / 4}$ and $5 e^{1 / 4} \mathrm{AM}$ in PS and IS, respectively. For the dot D1e a pairing of EAs in IS indicates $3 e^{2 / 7} \mathrm{AM}$, which corresponds to $B_{\mathrm{bi}} \sim 11 \mathrm{~T}$. For $B_{\mathrm{bi}} \sim 6 \mathrm{~T}$ of the dot D1d the PS state is $2 e^{3 / 5} \mathrm{AM}$.

In Fig. $3 \mathrm{f}$ we present expected schematic arrangements of $e^{v} \mathrm{~s}$ in these three AMs obtained by placing vortexes onto the maxima of their $e$ distribution. The $e^{3 / 5}$ and $e^{2 / 7}$ are realizations of the complex $e^{v}$ s, in which $v=n / p$ and $n>1$, consisting of $n$-es fused by a "coupling" vortex (see below). The figure accounts for different $e$ distribution size, i.e., $r_{\mathrm{s}}$, and demonstrates matching of $v$ and $r_{\mathrm{s}}$. This justifies our analysis, which, being extended to the rest of the dots, results in the values $v$ and $B_{\mathrm{bi}}$ and the $e^{v}$-AM configurations in the PS given in Table 1 . The analysis shows factor-of-two increase of $B_{\mathrm{bi}}$ for a $20 \%$ increase of $r_{\mathrm{s}}$ in agreement with the experiment (see Table 1 ).

Magneto-electrons and topological quantum computations. While the AM configurations observed and analyzed in $6 e$ states show symmetric, homogenous molecular structures compatible with distorted five- and sixfold circular symmetry and with the calculated electron density distributions, for $7 e$ state they are not. The observed anomalous 1D composite configuration for the $7 e$ state in the dot D1e (see Fig. $2 \mathrm{~d}$ ) can be assigned to a decomposition of a symmetrical $3 e^{2 / 7} \mathrm{AM}$ of a $6 e$ state after an extra $e$ is added. In this case, the additional $e^{2 / 7}$ needed for symmetrical arrangement, cannot be generated, since it requires pair of es. Thus, we can suppose that the additional $e$ creates $e^{1 / 3}$, which results to a transition from 21 to 24 vortexes state. The corresponding configuration could then consists of a $3 e^{1 / 3} \mathrm{AM}$ and a single $e^{4 / 15}$. The corresponding arrangements of the $e^{v}$ s are presented in Figs. $4 \mathrm{a}$,b. In the figures the coupling vortexes are shown by dashed circle.

Comparison of the arrangements and particle displacements in the scheme of Fig. 4a,b reveals transformations and interchange of $e^{v} \mathrm{~s}$, which correspond to elementary topological quantum computing operations (TQCOs). These involve first, two unfusions of $\left(e_{0}-e_{4}\right)$ and $\left(e_{3}-e_{5}\right) e^{2 / 7}$ anyon pairs resulting in four $e^{1 / 3}$ single anyons; second, two braids of $\left(e_{0}, e_{4}\right)$ and $\left(e_{1}, e_{3}\right)$ pairs; and, third, two fusions, which are a fusion of a $e^{1 / 3}\left(e_{3}, e_{6)}\right.$ pair forming a $e^{2 / 7}$ anyon and a fusion of a $e^{2 / 7}\left(e_{1}-e_{2}\right)$ and $a\left(e_{3}-e_{6}\right)$ pair forming $e^{4 / 15}$ anyon.

The above configuration transformation involves spatially separated $e^{v}$-anyons localized on the specific levels of QD confinement potential and a full description of such a transformation should specify a localization energy, 
i.e., include the energy coordinate. Along these lines we add an energy coordinate to $x-y$ plots and present the energy/space diagrams in Fig. 4c, d overlaid on the confinement potential. The diagrams outline a few meV difference in the confinement energy of coupled $e^{v}$.

They show that the coupling vortex should be presented by a pair of half-flux vortexes synchronously generated in gapped $e^{v}$-states. These vortexes should have twice size (not shown in the figure) and thus embrace one of the neighboring vortexes. While such a representation of complex $e^{v}$ s seems unusual in the framework of conventional theories of FQHE states, assuming degenerate states and zeros of many-electron wave-functions for the vortex description ${ }^{34}$, it is supported by our data and by the experimental observations of $2 / 5$ anyons by Aharonov-Bhom interferometry in quantum Hall bars ${ }^{48}$, giving independent evidence of their existence.

TQCOs resulting from the PL process in the dot D1e, presented in the Fig. $4 \mathrm{a}, \mathrm{b}$, are described by a $B_{7}$ braid group diagram, i.e., time-position world lines, shown in Fig. $4 \mathrm{e}$. The braid of $e_{4}-e_{0}$ and $e_{4}-e_{0} e^{1 / 3}$-anyons should result in a $\Pi / 3$ phase change ${ }^{23}$, which according to the diagram, is encoded in the unfusion of the corresponding $e^{2 / 7}$ anyon and accompanied by a local charge redistribution. This allows one to use local charge sensing to control TQCOs, which can be provided with high sensitivity by a single-e transistor (SET) ${ }^{49}$. Since control of phase is a key step in TQCOs and a technology and methodology of SET fabrication and measurements are well developed $^{50}$, using SETs opens up a new possibility for physical realization of TQGs.

The design of the TQG based on InP/GaInP2 $e^{v}$-AMs will include few SETs and nano-electrode gates having sizes $\sim 50 \mathrm{~nm}$ adjacent to individual QDs formed by semiconductor manufacturing technology using $e$-beam lithography ${ }^{51}$. The arrays of QDs (see Fig. 2e)] can be used to realize circuits. Since we demonstrated that $e^{v}$-AM structure is sensitive to small variation of $r_{s}$, using nano-gates changing local potentials near QD gives possibility of the fine tuning of the initial state.

In Fig. 4a,b we draw three SETs and a gate near D1e QD, which can be used to realize a prototype of the device for electrical testing of TQCOs shown in Fig. 4e. In the device RF SETs acting simultaneously and utilizing a single RF line by means of carrier multiplexing ${ }^{52}$ will be used to detect "charge triangulation". Each of the devices is biased at the slope of SET transfer characteristic and employs charge cancellation technique ${ }^{53}$ to minimize effects of direct capacitive coupling to the pulsing gate. In this way signals obtained from SETs (Us $\left.{ }^{i}\right)$ under variation of $r_{\mathrm{s}}$ or injection/removing of the electrons by the gate voltage $\left(U_{\mathrm{G}}\right)$, i.e., $U \mathrm{~s}^{i}\left(U_{\mathrm{G}}\right)$-functions, will be used for designing of topological quantum computing processing.

Finally, we should point out, that the QDs considered do not directly involve NA anyons and MZMs, as suggested in initial TQC proposals. However, our magneto-PL measurements and the preliminary analysis of $N \sim 8 \mathrm{InP} / \mathrm{GaInP}_{2}$ dots having $r_{\mathrm{s}} \sim 1.5$ (similar to D04 in the insert in Fig. 1a), which will be published elsewhere, reveal $B_{\mathrm{bi}} \sim 2$ T close to $v \sim 5 / 2$. This can indicate $e^{1 / 4}$ anyon supporting MZMs, similar to that discussed for corresponding SLL FQHE state. The possibility of forming of the corresponding state in an appropriate QD naturally follows from $e^{v}-E_{\text {tot }}$ of in Fig. 3e for $v>1$. Moreover, for such dot types we also observed a quasi 1D shape of EA ${ }^{36}$, similar to that in Fig. 2b, which can be a signature of TQCOs. We already demonstrated Coulomb blockade control of $N$ in these dots in the range 8-18 having $\Delta U_{\mathrm{G}} \sim 0.3 \mathrm{meV}$ per electron and the possible formation of a spin-polarized state for $N>15^{54}$.

\section{Conclusion}

We have introduced a novel system, which can be used for the realization of TQG. These are $e^{v}$-anyon molecules $\left(e^{v}-\mathrm{AM}\right)$ naturally formed in self-organized many electron InP/GaInP 2 QD structures. We demonstrated their promising TQC prospects using high-spatial-resolution magneto-PL measurements of five-to-seven electron QDs having $r_{\mathrm{s}} \sim 2$. Our data, together with the quantum mechanical calculations of their electronic structure in a magnetic field and analysis demonstrate a self-formation of $e^{v}$ AMs, having $v \sim 3 / 5-1 / 4$, corresponding to a built-in magnetic field of 6-12 T. Our measurements of PL spectra and the mapping of the intensity of individual PL lines gave a direct imaging of molecular configurations and allow the observation of a transformation of a $e^{v}$ arrangement in a $e^{2 / 7}-\mathrm{AM}$ under photo-excitation involving fusion and braiding of the anyons. The observed transformation reveals a significant redistribution of the fractional charge within the dot, which suggests the use of single electron transistor charge sensing to control TQC operations. Our investigations show that InP/GaInP $\mathrm{P}_{2}$ AM QDs having intrinsic anyon localization at zero external magnetic field combined with charge sensing control of anyon's states open up novel directions for the realization of TQC.

\section{Methods}

Magneto-PL measurements and data processing. Spatially-resolved magneto-PL spectra were measured using NSOM operating at $10 \mathrm{~K}$ and magnetic fields of up to $10 \mathrm{~T}$ and using optical fiber probes having an aperture size of 50-300 $\mathrm{nm}$ in a collection-illumination mode. The spectra were excited by the $514.5 \mathrm{~nm}$ Ar-laser line and measured using a CCD (multi-channel) detector together with a $280 \mathrm{~mm}$ focal length monochromator. The excitation power measured before the fiber coupler was $\sim 5 \mathrm{~W}$, which provided a power density of $\sim 0.5 \mathrm{~W} /$ $\mathrm{cm}^{2}$. The spectral resolution of the system is $0.2-0.4 \mathrm{meV}$.

For the Lorenz contour deconvolution of PL spectra, we used a multi-peak fitting procedure from Origin 8.0 graphic software.

The spatially-resolved PL intensity at the selected wavelengths (image) was generated using the spectra taken in a square grid having a mesh of $50 \mathrm{~nm}$. We plotted the experimental data using a contour plot option of Origin 8.0 and the division of the intensity data into 20 levels. The size of the emission area was estimated as a size of a NSOM image at the emission intensity level of 0.9 , i.e. two upper contour plot levels. NSOM image of ASC are related to PS Stokes components images correspond to a difference of electron positions in the IS and PS ${ }^{36}$. 
Configuration interaction and Hartree-Fock many-body calculations. The states of $N$ confined electrons ( $N \sim 6$ and $\left.r_{\mathrm{s}} \sim 2.5\right)$ in a strong perpendicular magnetic field $B_{z}=B$ are modeled theoretically by configuration interaction (CI), using a method described in more detail in Ref. ${ }^{36}$. We assume a quasi-2D effectivemass approximation with Hamiltonian (in atomic units, $4 \pi \epsilon_{0}=m_{e}=\hbar=|e|=1$ )

$$
H=\sum_{i=1}^{N}\left\{\frac{1}{2 m^{*}}\left[\mathbf{p}_{i}-e \mathbf{A}\left(\mathbf{r}_{i}\right)\right]^{2}+V_{\mathrm{ext}}\left(\mathbf{r}_{i}\right)+g^{*} \mu_{B} B_{z} S_{z i}\right\}+\sum_{i>j}^{N} \frac{e^{2}}{\kappa\left|\mathbf{r}_{i}-\mathbf{r}_{j}\right|},
$$

where the magnetic vector potential is given by $\mathbf{A}(x, y)=\left(B_{z} / 2\right)(-y, x, 0)$, and the effective mass $m^{*}=0.077$ and dielectric constant $\kappa=12.61$ are taken to be the values for bulk InP. The effective $2 \mathrm{D}$ confining potential $V_{\text {ext }}(\mathbf{r})$ in the $x-y$ plane is assumed to be harmonic near the center, with frequency parameter $\hbar \omega_{0}$, and to develop a "hard wall" near the physical boundary of the dot on the substrate. The confining potential also has a slight angular deformation, indicated by the potential contours in Fig. 3a, which was chosen to be typical of the dots synthesized experimentally. The spin Zeeman term $g^{*} \mu_{B} B_{z} S_{z i}$ is usually not included explicitly. The first step in the CI calculation is to calculate a single-particle basis set $|i\rangle$ using spin-polarized Hartree-Fock (HF),

$$
\left[\frac{1}{2 m^{*}}(\boldsymbol{p}-e \mathbf{A})^{2}+V_{\mathrm{ext}}+V_{\mathrm{HF}}\right]|i\rangle=\epsilon_{i}|i\rangle .
$$

The lowest $N$ HF orbitals $|a\rangle$ (where $a=1, \ldots, N$ ) are occupied, with one electron per orbital assuming complete spin polarization. The occupied orbitals contribute to the self-consistent HF potential $V_{\mathrm{HF}}$, which is defined as

$$
\left\langle j\left|V_{\mathrm{HF}}\right| i\right\rangle=\frac{e^{2}}{\kappa} \sum_{a}^{\text {occ }}\left(\left\langle j a\left|r_{12}^{-1}\right| i a\right\rangle-\left\langle j a\left|r_{12}^{-1}\right| a i\right\rangle\right),
$$

where $|i\rangle$ and $|j\rangle$ are general states (occupied or unoccupied). The eigenvalues $\epsilon_{a}$ of the occupied orbitals, at least for zero to moderate $B_{z}$, may be expected to give approximations to the removal energies of electrons from the system, according to Koopman's theorem from atomic and molecular physics ${ }^{54}$. In the calculated electron density distributions the dot size $\left(D_{c}\right)$ was estimated as the size of the area containing $96 \%$ of electron density.

Received: 25 June 2021; Accepted: 18 October 2021

Published online: 02 November 2021

\section{References}

1. Kitaev, A. Y. Fault-tolerant quantum computation by anyons. Ann. Phys. 303, 2-30 (2003).

2. Freedman, M. H. P/NP, and the quantum field computer. PNAS 95, 98-101 (1998).

3. Bonesteel, N. E., Hormozi, L., Zikos, G. \& Simon, S. H. Braid topologies for quantum computation. Phys. Rev. Lett. 95, 140503140504 (2005).

4. Sarma, S. D., Freedman, M. \& Nayak, C. Topologically protected qubits from a possible non-abelian fractional quantum Hall state. Phys. Rev. Lett. 94, 166802-166804 (2005).

5. Mourik, V. et al. Signatures of Majorana fermions in hybrid superconductor-semiconductor nanowire devices. Science 336, 10031007 (2012).

6. Nadj-Perge, S. et al. Observation of Majorana fermions in ferromagnetic atomic chains on a superconductor. Science 346, 602-6007 (2012).

7. Xu, J.-P. et al. Experimental detection of a Majorana mode in the core of a magnetic vortex inside. Phys. Rev. Lett. 114, 017001017005 (2015).

8. He, Q. L. et al. Chiral Majorana fermion modes in a quantum anomalous Hall insulator-superconductor structure. Science 357, 294-299 (2017).

9. Kasahara, Y. et al. Majorana quantization and half-integer thermal quantum Hall effect in a Kitaev spin liquid. Nature 559, 227-231 (2008).

10. Sarma, S. D., Freedman, M. \& Nayak, C. Majorana zero modes and topological quantum computation. npj Quantum Inf. 1, 15001-15013 (2015).

11. Wan, X., Hu, Z.-X., Rezayi, E. H. \& Yang, K. Fractional quantum Hall effect at $v 1 / 4$ 5=2: Ground states, non-Abelian quasiholes, and edge modes in a microscopic model. Phys. Rev. B 77, 165316-165415 (2008).

12. Prodan, E. \& Haldane, F. D. M. Mapping the braiding properties of the Moore-Read state. Phys. Rev. B 80, 115121-115214 (2009).

13. Macaluso, E., Compari, T., Mazza, L. \& Carusotto, I. Fusion channels of non-abelian anyons from angular-momentum and densityprofile measurements. Phys. Rev. Lett. 123, 266801-266807 (2019).

14. Ladd, T. D. et al. Quantum computers. Nature 464, 45-53 (2010).

15. Shor, P. W. Scheme for reducing decoherence in quantum computing memory. Phys. Rev. A 52, R2493-R2496 (1995).

16. Barends R.et al,. Superconducting quantum circuits at the surface code threshold for fault tolerance. Nature 508, 500-503 (2014).

17. Harty, T. P. et al. High-fidelity preparation, gates, memory, and readout of a trapped-ion quantum bit. Phys. Rev. Lett. 113, 220501-220505 (2014).

18. Veldhorst, M. et al. An addressable quantum dot qubit with fault-tolerant control-fidelity. Nat. Nanotechnol. 9, 981-985 (2014).

19. Watson, F. et al. A programmable two-qubit quantum processor in silicon. Nature 555, 633-637 (2015).

20. He, Y. et al. A two-qubit gate between phosphorus donor electrons in silicon. Nature 571, 371-375 (2019).

21. Rong, X. et al. Experimental fault-tolerant universal quantum gates with solid-state spins under ambient conditions. Nat. Commun. 6, 8748-8757 (2015).

22. Wright, K. et al. Benchmarking an 11-qubit quantum computer. Nat. Commun. 10, 5464-5466 (2019).

23. Wilczek, F. Magnetic flux, angular momentum, and statistics. Phys. Rev. Lett. 48, 1144-1146 (1982).

24. Moore, G. \& Read, N. Nonabelions in the fractional quantum Hall effect. Nucl. Phys. B 360, 362-396 (1991).

25. Volovik, G. E. Fermion zero modes on vortices in chiral superconductors. JETP Lett. 70, 609-614 (1999).

26. Read, N. \& Green, D. Paired states of fermions in two dimensions with breaking of parity and time-reversal symmetries and the fractional quantum Hall effect. Phys. Rev. B 61, 10267-10297 (2000). 
27. Ivanov, D. A. Non-abelian statistics of half-quantum vortices in p-wave superconductors. Phys. Rev. Lett. 86, 268-271 (2001).

28. Tsui, D. C., Stormer, H. L. \& Gossard, A. C. Two-dimensional magneto-transport in the extreme quantum limit. Phys. Rev. Lett. 48, 1559-1562 (1982).

29. Xia, J. S. et al. Electron correlation in the second Landau level: A competition between many nearly degenerate quantum phases. Phys. Rev. Lett. 93, 176809-176814 (2004).

30. Choi, H. C., Kang, W., DasSarma, S., Pfeiffer, L. N. \& West, K. W. Activation gaps of fractional quantum Hall effect in the second Landau level. Phys. Rev. B 77, 081301-4(R) (2008).

31. Dolev, M., Heiblum, M., Umansky, V., Stern, A. \& Mahalu, D. Observation of a quarter of an electron charge at the v=5/2 quantum Hall state. Nature 452, 829-834 (2008).

32. Willett, R. L., Nayak, C., Shtenge, K., Pfeiffer, L. N. \& West, K. W. Magnetic-field-tuned Aharonov-Bohm oscillations and evidence for non-abelian anyons at $\mathrm{v}=5 / 2$. Phys. Rev. Lett. 111, 186401-186405 (2013).

33. Banerjee, M. et al. Observation of half-integer thermal Hall conductance. Nature 559, 205-210 (2018).

34. Laughlin, R. B. Anomalous quantum Hall effect: An incompressible quantum fluid with fractionally charged excitations. Phys. Rev. Lett. 50, 1395-1398 (1983).

35. Mintairov, A. M. et al. Fractional charge states in the magneto-photoluminescence spectra of a single-electron quantum dot. Nanomaterials 11, 493-514 (2021).

36. Mintairov, A. M. et al. Control of Wigner localization and electron cavity effects in near-field emission spectra of In(Ga)P/GaInP quantum-dot structures. Phys. Rev. B 97, 195443-195449 (2018).

37. Mintairov, A. M., Merz, J. L. \& Blundell, S. Molecular states of electrons: emission of single molecules in self-organized InP/GaInP quantum dots. in Fingerprints in the Optical and Transport Properties of Quantum Dots, InTech ISBN 978-953-51-0648. 126-152 (2012).

38. Mintairov, A. M., Kapaldo, J., Merz, J. L., Vlasov, A. S. \& Blundell, S. A. Wigner molecules and charged excitons in near-field magnetophotoluminescence spectra of self-organized InP/GaInP2 quantum dots. Phys. Rev. B 95, 11544-11610 (2017).

39. Mintairov, A. M. et al. Nanoindentation and near-field spectroscopy of single semiconductor quantum dots. Phys. Rev. B 69, 155306-155312(2004).

40. Chacraborty, T. Physics of artificial atoms: Quantum dots in a magnetic field. Comments Condens. Matter Phys. 16, 35-68 (1992).

41. Maksym, P. A., Imamura, H., Mallon, G. P. \& Aoki, H. Molecular aspects of electron correlation in quantum dots. J. Phys. Condens. Matter 12, R299-R334 (2000).

42. Reimann, S. M. \& Manninen, M. Electronic structure of quantum dots. Rev. Mod. Phys. 74, 1287-1343 (2002).

43. Jacak, L., Hawrylak, P. \& Wojs, A. Quantum Dots 176 (Springer, 1998).

44. Phannkuche, D., Gernhardts, R. R., Maksym, P. A. \& Gudmundson, V. Theory of quantum dot helium. Physica B 189, 6-15 (1993).

45. Ruan, W. Y., Liu, Y. Y., Bao, C. G. \& Zhang, Z. Q. Origin of magic angular momenta in few-electron quantum dots. Phys. Rev. B 51, 7942-7945 (1995).

46. Yannouleas, C. \& Landman, U. Unified microscopic approach to the interplay of pinned-Wigner-solid and liquid behavior of the lowest Landau-level states in the neighborhood of $v=1 / 3$. Phys. Rev. B 84, 165327-165417 (2011).

47. Reimann, S. M., Koskinen, M., Manninen, M. \& Mottelson, B. R. Quantum dots in magnetic fields: Phase diagram and broken symmetry at the maximum-density-droplet edge. Phys. Rev. Lett. 83, 3270-3273 (1999).

48. Venkatachalam, V., Yacoby, A., Pfeiffer, L. \& West, K. Local charge of the $v=5 / 2$ fractional quantum Hall state. Nature 469, 185-188 (2011).

49. Aassime, A., Johansson, G., Wendin, G., Schoelkopf, R. J. \& Delsing, P. Radio-frequency single-electron transistor as readout device for qubits: Charge sensitivity and backaction. Phys. Rev. Lett. 86, 3376-3379 (2001).

50. Crippa, A. et al. Electrical spin driving by g-matrix modulation in spin-orbit qubits. Phys Rev. Lett. 120, 137702-137705 (2018).

51. Stevenson, T. R., Pellerano, F. A., Stahle, C. M., Aidala, K. \& Schoelkopf, R. J. Multiplexing of radio-frequency single-electron transistors. Appl. Phys. Lett. 80, 3012-3014 (2002).

52. Yong, T., Orlov, A. O., Snider, G. L. \& Patrick, F. J. Radio frequency operation of clocked quantum-dot cellular automata latch. Appl. Phys. Lett. 95, 193109-193113 (2009).

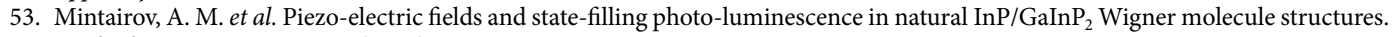
Appl. Phys. Lett. 118, 121101 (2021).

54. Lindgren, I. \& Morrison, J. Atomic Many-Body Theory 2nd edn. (Springer, 1986).

\section{Acknowledgements}

A.M.M., D.V.L. and A.S.V acknowledge the financial support of the Russian Science Foundation (project \#1919-00246) of magneto-PL NSOM experiments. G.S., A.O. and A.M.M. acknowledges the financial support of the National Science Foundation Grant (\#DMR-1904610) of development TQG proposal.

\section{Author contributions}

A.M.M., D.V.L and A.S.V. conceived the magneto-PL NSOM experiment. G.L.S. and A.O.O. suggest and describe the proposal for TQG using SET. The CI and HF calculations were carried out by S.A.B., using codes developed by him. The manuscript was written by A.M.M. with the input from all authors and with the critical contributions from S.A.B., A.O.O., and G.L.S.

\section{Competing interests}

The authors declare no competing interests.

\section{Additional information}

Correspondence and requests for materials should be addressed to A.M.M.

Reprints and permissions information is available at www.nature.com/reprints.

Publisher's note Springer Nature remains neutral with regard to jurisdictional claims in published maps and institutional affiliations. 
(c) (i) Open Access This article is licensed under a Creative Commons Attribution 4.0 International cc) License, which permits use, sharing, adaptation, distribution and reproduction in any medium or format, as long as you give appropriate credit to the original author(s) and the source, provide a link to the Creative Commons licence, and indicate if changes were made. The images or other third party material in this article are included in the article's Creative Commons licence, unless indicated otherwise in a credit line to the material. If material is not included in the article's Creative Commons licence and your intended use is not permitted by statutory regulation or exceeds the permitted use, you will need to obtain permission directly from the copyright holder. To view a copy of this licence, visit http://creativecommons.org/licenses/by/4.0/.

(C) The Author(s) 2021 